Pesq. Vet. Bras. 36(1):55-61, janeiro 2016 DOI: $10.1590 / \mathrm{S} 0100-736 \mathrm{X} 2016000100009$

\title{
Parâmetros reprodutivos e produtivos em vacas leiteiras de manejo free stall ${ }^{1}$
}

\begin{abstract}
Faider A.C. Villadiego ${ }^{2 *}$, Jhonata V. Pereira ${ }^{2}$, Eduardo P. da Costa ${ }^{3}$, Marcos I. Marcondes ${ }^{4}$, Victor E.G. Leon ${ }^{2}$, Paula P. Maitan², Amanda R. Nogueira ${ }^{2}$ e José D. Guimarães ${ }^{3}$

ABSTRACT.- Villadiego F.A.C., Pereira J.V., Guimarães J.D., Costa E.P., Marcondes M.I., Leon V.E.G., Maitan P.P. \& Nogueira A.R. 2016. [Productive and reproductive parameters of dairy cows in free stall.] Parâmetros reprodutivos e produtivos em vacas leiteira de manejo free stall. Pesquisa Veterinária Brasileira 36(1):55-61. Setor de Reprodução e Produção Animal, Universidade Federal de Viçosa, Av. Peter Henry Rolfs s/n, Campus Universitário, Viçosa, MG 36570-000, Brazil.E-mail: faider_cas@hotmaill.com

The reproductive and productive parameters in postpartum of 51 primiparous and pluriparous Holstein cows housed in free stall were obtained. The values measured were: body condition score at parturition $(3.47 \pm 0.4)$, interval from parturition to first ovulation (5.89 \pm 3.4 weeks), interval parturition at first service $(112.5 \pm 57.0$ days), first-service pregnancy rate $(43.6 \%)$ and during the experimental period (76.4\%). No difference was found between parturition order $(\mathrm{P}>0.05)$. The accumulated milk production until conception time, daily production until conception time, and adjusted 305-day milk production was respectively $3,335.6 \pm 1,488.4 ; 25.8 \pm 5.4$ and $7,424.0 \pm 1,877.8$ liters. In free stall of this study the estrus detection was the most limiting factor to improve the reproductive efficiency.

INDEX TERMS: Free stall, immediate postpartum, cyclical ovarian activity, fertility.
\end{abstract}

RESUMO.- Avaliou-se parâmetros reprodutivos e produtivos em vacas da raça Holandês-PB no pós-parto imediato, manejadas em free stall. Utilizou-se 51 vacas entre primíparas e pluríparas. Os valores obtidos foram: escore de condição corporal ao parto $3,47 \pm 0,4$; intervalos do parto à primeira ovulação $5,89 \pm 3,4$ semanas; ao primeiro serviço $112,5 \pm 57,0$ dias; a taxa de gestação ao primeiro serviço foi $43,6 \%$ e no período experimental $76,4 \%$, não sendo observada diferença para ordens de parto $(\mathrm{P}>0,05)$. A produção de leite acumulada à concepção, produção diária leite até a concepção e produção de leite ajustado para 305 foi respectivamente, $3.335,6 \pm 1.488,4 ; 25,8 \pm 5,4$ e $7.424,0 \pm 1.877,8$ litros. Conclui-se que no manejo de Free stall, a detecção de estro, foi o principal fator limitante para alcançar melhor eficiência reprodutiva.

\footnotetext{
${ }^{1}$ Recebido em 22 de abril de 2015.

Aceito para publicação em 24 de novembro de 2015.

${ }^{2}$ Pós-Graduação em Medicina Veterinária, Laboratório de Reprodução Animal, Universidade Federal de Viçosa (UFV), Avenida Peter Henry Rolfs s/n, Campus Universitário, Viçosa, MG 36570-000, Brasil. *Autor para correspondência: faider_cas@hotmail.com

${ }^{3}$ Docente (Coordenador) do Departamento de Medicina Veterinária, Área de Reprodução e Produção Animal, UFV, Viçosa, MG 36570-000, Brasil.

${ }^{4}$ Docente do Departamento de Zootecnia, Área de Nutrição em Gado de Leite, Universidade Federal de Viçosa, MG 36570-000, Brasil.
}

TERMOS DE INDEXAÇÃO: Atividade ovariana cíclica, fertilidade, free stall, pós-parto imediato.

\section{INTRODUÇÃO}

O puerpério compreende o período entre o parto e o aparecimento do primeiro estro compatível com o estabelecimento de uma nova gestação (Marques Júnior 1993, Horta 1995). De acordo com Short et al. (1990), o restabelecimento da função reprodutiva no pós-parto depende da involução uterina normal e o restabelecimento dos feedback no eixo hipotalâmico-hipofisário-gonadal, e consequente retorno a ciclicidade.

O LH (Hormônio Luteinizante) e não o FSH (Hormônio Folículo Estimulante) é o hormônio limitante para o início da atividade ovariana pós-parto (Yavas \& Walton 2000). Depois que os estoques de LH são restabelecidos entre 15 e 30 dias no pós-parto, secreções de estradiol $\left(E_{2}\right)$, eventualmente, atingem limares que ativam a liberação de picos de GnRH, e consequentemente causam maior amplitude do pico de liberação do LH, desencadeando o processo de ovulação.

Em bovinos de aptidão leiteira, um retorno precoce a atividade ovariana é importante para que se obtenha maior número de concepções no início da lactação. Assim, o período de espera voluntária é de 45 a 60 dias. Quanto antes 
ocorrer a primeira ovulação no pós-parto, maior será o número de ciclos estrais durante esse período de espera voluntária. Tanto a expressão de sinais de estro quanto à fertilidade aumentam com o número de ciclos estrais antes da primeira inseminação artificial pós-parto (Santos 2005).

Tendo em vista a influência da involução uterina, o puerpério e à condição corporal sobre ao retorno da atividade ovariana e a performance reprodutiva. Objetivou-se no presente estudo, avaliar parâmetros reprodutivos e produtivos em vacas da raça Holandês-PB no pós-parto imediato, mantidos em manejo de free stall.

\section{MATERIAL E MÉTODOS}

0 experimento foi conduzido em uma granja leiteira localizada, 660 metros de altitude, no município de Viçosa, Minas Gerais, Brasil, altitude de 660 metros, $20^{\circ}, 45^{\prime}, 16,3^{\prime \prime}$ latitude sul $42^{\circ}, 52^{\prime}$, 57,02 ", e longitude Oeste de Greenwinh, temperatura média anual de $20,9^{\circ} \mathrm{C}$. Os procedimentos experimentais utilizando animais foram aprovados pela Comissão de Ética da Universidade Federal de Viçosa (CEUA/UFV, processo №27/2012, em 26.06.2012).

Foram utilizadas 51 vacas, sendo 13 primíparas e 38 pluríparas da raça Holandês-PB, mantidas em manejo de free stall, com idade entre 2,5 e 7 anos e peso corporal médio de 594,4 $\pm 65,1 \mathrm{~kg}$. 0 período experimental foi de janeiro/2011 a maio/2012.

Os animais foram alimentados duas vezes ao dia, com silagem de milho e concentrado, em cochos de concreto abrigados sob a cobertura dos galpões freestall, onde os animais permaneceram durante todo o período de lactação. A dieta das vacas no pós-parto de 0 a 20 dias pós-parto foi composta por silagem de milho e concentrado, sendo $6 \mathrm{~kg}$ de concentrado/vaca/dia e silagem de milho a vontade até completar 20 dias de lactação, a relação cátion da dieta foi controlada para ficar constante em mais de 5 meq/100gMS. Para os animais de 20 a 80 dias em lactação, receberam $12 \mathrm{~kg}$ de concentrado/vaca/dia, com a energia suficiente para produzir $42 \mathrm{~kg} / \mathrm{vaca} /$ dia. Para os animais com mais de 80 dias em lactação,foi fornecido $1 \mathrm{~kg}$ de concentrado para cada $3 \mathrm{~kg}$ de leite/dia, para o animal que produzia mais de $25 \mathrm{~kg}$ de leite/dia e $1 \mathrm{~kg}$ para 4,5 kg de litro/dia para vacas que produzia menos de $25 \mathrm{~kg}$ de leite/dia, e silagem de milho foi fornecida a vontade para todos os animais, conforme o manejo adotado na granja. Todas as dietas foram feita de acordo com NRC (2001).

Todos os animais foram pesados no primeiro dia pós-parto e depois semanalmente até o dia da inseminação artificial em uma balança individual e avaliados segundo o escore de condição corporal (ECC), de acordo com os procedimentos empregados por Ferreira (1900). Na avaliação de ECC, se utilizou a escala de 1 a 5 (1= muito magra e $5=$ muito gorda), com a inclusão de 0,25 pontos. Os animais, após as pesagens, foram avaliados de acordo com a deposição de gordura na região das costelas, tuberosidade isquiática, tuberosidade ilíaca, dorso e ao redor da inserção da cauda, para posterior atribuição do escore sendo os procedimentos realizados por dois avaliadores treinados.

A detecção de estro foi feita por pessoal treinado previamente, realizado duas vezes ao dia, às 7 hora da manha e às 17 horas, por meia hora de observação visual. 0 momento ideal para inseminação foi determinado de acordo com o protocolo de inseminação de Trimberg \& Davis (1943), de modo que as fêmeas observadas em estro à tarde foram inseminadas na manhã do dia seguinte (7 às $8 \mathrm{~h}$ ) e aquelas em estro pela manhã foram inseminadas no final da tarde do mesmo dia (18 às 19h).

Os exames ultrassonográficos foram realizados por via transretal, utilizando um aparelho portátil da marca CHISON ${ }^{\circledR}$, modelo D600 VET acoplado a um transdutor linear de $7 \mathrm{MHz}$. 0 retorno da atividade ovariana foi avaliado a partir de 15 dias pós-parto, verificando-se o histórico ou alguma ocorrência de patologias como retenção de placenta ou infecção uterina. Por meio de exames ultrassonograficos foi avaliado o desenvolvimento folicular semanalmente, onde foi computado o número de folículos e diâmetros do maior e do segundo maior folículo presente em cada ovário. Esse procedimento foi realizado até a confirmação da gestação. Ou após a inseminação o animal não era mais avaliado semanalmente e somente no dia do diagnóstico de gestação.

0 diagnóstico de gestação foi realizado de 28 a 35 dias após IA, por avaliação ultrassonográfica, sendo considerada fêmea gestante, aquela com presença do liquido amniótico e embrião. As vacas gestantes foram retiradas do experimento e as não gestantes permaneciam até que fossem diagnosticadas como gestantes ou no final do período experimental (maio/2012).

Para analise dos dados obtidos no experimento foi empregado o programa estatístico SAEG-9.1 (UFV, 2007). Para as variáveis qualitativas, os dados foram arranjados em tabela de contingência e analisados pelo teste de qui-quadrado, com $5 \%$ de probabilidade de erro. Para as variáveis quantitativas foi efetuado o teste de Lilliefors e Cochran e Bartlett para verificar a normalidade da distribuição dos dados e a homogeneidade das variâncias, respectivamente, e posteriormente submetidas a ANOVA, com a comparação das médias pelo teste de Tukey, com $5 \%$ de probabilidade de erro. Quando não atendida as premissas da ANOVA, os dados foram avaliados pela análise não paramétrica, com as médias comparadas pelos testes de Kruskal Wallis ou Wilcoxon, com 5\% de probabilidade de erro.

\section{RESULTADOS E DISCUSSÃO}

0 escore de condição corporal médio ao parto foi de $3,47 \pm 0,4$, e o peso corporalmédio ao parto foi de $594,4 \pm 65,1$ $\mathrm{kg}$, Ambos não diferiram $(\mathrm{P}>0,05)$ entre as ordens de parto (Quadro 1). 0 valor de referência para o peso corporal ao parto de novilhas da raça Holandês-PB é de aproximadamente $500 \mathrm{~kg}$, correspondendo aos $80 \%$ do peso adulto dessa raça (Campos \& Lizieire 1998), o que não foi observado no presente estudo, para as novilhas da mesma raça foi de $564,2 \pm 80,9 \mathrm{~kg}$, correspondente a $91,3 \%$ do peso do rebanho adulto, indicando o melhoramento genético dos animais e o bom manejo nutricional no qual as fêmeas foram submetidas durante a fase de cria e recria.

Neste estudo o ECC médio ao parto, para todos os ani-

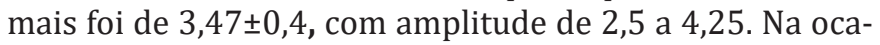
sião do parto em bovinos de aptidão leiteira recomenda-se que o ECC ao parto seja de 3,0 a 4,0, visto que, a obesidade ou baixa ECC ao parto pode aumentar a ocorrências de doenças puerperais, e aumentando o período de anestro pós-parto, e ocasionar redução da taxa de gestação (Butler

Quadro 1. Peso corporal (PESPART) e escore de condição corporal (ECCPART) ao parto em vacas da raça Holandesa-PB, criadas em regime de free stall, de acordo com a ordem do parto (ORDPART)*

\begin{tabular}{ccc}
\hline ORDPART & PESPART (kg) & ECCPART (escala de 1 a 5) \\
\hline 1 & $564,2 \pm 80,9$ & $3,55 \pm 0,4$ \\
2 & $590,5 \pm 58,9$ & $3,46 \pm 0,4$ \\
3 & $607,0 \pm 38,8$ & $3,34 \pm 0,4$ \\
4 & $659,7 \pm 71,1$ & $3,75 \pm 0,8$ \\
5 & $616,0 \pm 33,6$ & $3,25 \pm 0,6$ \\
Geral & $594,4 \pm 65,1$ & $3,47 \pm 0,4$
\end{tabular}

* $(\mathrm{P}>0,05)$ pelo teste de Tukey. 
2006); já, o mesmo autor recomenda para vacas de alta produção de leite, escores ao parto esteja compreendido entre 3 e 3,25 (uma escala 1 a 5).

No presente estudo, independente do período da lactação, vacas primíparas apresentaram maior ECC, quando comparadas as vacas pluríparas $(\mathrm{p}<0,05)$. 0 ideal é que as vacas apresentassem boa condição corporal ao final da lactação, já próxima ao parto subsequente, uma vez que, a condição corporal durante o período seco pode afetar negativamente a saúde ao parto. Vacas obesas à secagem, ao parto e no início da lactação apresentam maior percentual de doenças no periparto, quando comparadas às vacas com bom ECC (Gearhart et al. 1990). No período seco, os animais devem manter o ECC de 3,0 a 3,5 visando diminuir a ocorrência de distócia, cistos ovarianos, transtornos reprodutivos e alterações locomotora após o parto, sendo estes os transtornos responsáveis pelo descarte involuntário (Butler 2006).

Neste estudo, o acompanhamento dos transtornos reprodutivos puerperais tais como: retenção de placenta, infecção uterina, cistos das vacas primíparas e pluríparas foram diagnosticados e tratados precocemente. Assim mesmo, em nenhuma das ordens de parição apresentaram animais em anestro pós-parto, e todos os animais do presente estudo apresentaram boa ECC ao parto.

Os parâmetros reprodutivos utilizados para analisar a eficiência reprodutiva no presente estudo, foram: intervalos de parto à primeira ovulação, ao primeiro serviço ou IA; taxa de gestação ao primeiro serviço; número de serviços por concepção e período de serviço.

A média de intervalo do parto a primeira ovulação foi de 5,89 $\pm 3,4$ semanas no pós-parto, sendo que $92 \%(12 / 13)$ das vacas primíparas ovularam pela primeira vez nos primeiros 42 dias pós-parto e 68\% (26/38) das vacas pluríparas, apresentaram o primeiro estro ovulatório até os 50 dias pós-parto. 0 ECC foi de $3,18 \pm 0,5$ à primeira ovulação, não sendo observada diferença $(\mathrm{P}>0,05)$ entre as ordens de parto (Quadro 2). Os animais não perderam, mas de 0.5 de ECC, entre ECC ao parto $(3,47 \pm 0,4)$ (Quadro 1 ), ECC a primeira ovulação $(3,18 \pm 0,5)$ e ECC a primeira inseminação artificial $(3,34 \pm 0,5)$ (Quadro 2).

A avaliação ultrassonográfica e dosagem da concentração de progesterona;no estudo de Vanholder et al., (2005) verificou respectivamente $63,4 \%$ dos animais ciclando normalmente até 50 dias e $64,7 \%$ dos animais haviam ovulado até 60 dias pós-parto.

Quadro 2. Intervalo do parto (IPOVU), ECC à primeira ovulação (ECC10VU), Intervalo do parto à primeira inseminação (IP1IA), ECC ao primeiro serviço (ECC1IA) e período de serviço de vacas da raça Holandesa-PB, manejadas em free stall de acordo com a ordem de parto (ORDPART)*

\begin{tabular}{cccccc}
\hline ORDPART & $\begin{array}{c}\text { IP1OVU } \\
\text { (semanas) }\end{array}$ & $\begin{array}{c}\text { ECC1OVU } \\
\text { (Escala 1 a 5) }\end{array}$ & $\begin{array}{c}\text { IP1IA } \\
\text { (dias) }\end{array}$ & $\begin{array}{c}\text { ECC1IA } \\
\text { (Escala 1 a 5) }\end{array}$ & $\begin{array}{c}\text { PSER } \\
\text { (dias) }\end{array}$ \\
\hline 1 & $4,41 \pm 4,5$ & $3,33 \pm 0,4$ & $117,8 \pm 64,0$ & $3,45 \pm 0,4$ & $182,7 \pm 101,8$ \\
2 & $6,35 \pm 3,4$ & $3,22 \pm 0,6$ & $112,0 \pm 54,9$ & $3,43 \pm 0,5$ & $161,6 \pm 85,4$ \\
3 & $6,12 \pm 2,4$ & $3,03 \pm 0,4$ & $90,2 \pm 46,8$ & $3,12 \pm 0,3$ & $160,6 \pm 114,0$ \\
4 & $7,25 \pm 2,4$ & $3,25 \pm 0,8$ & $101,2 \pm 42,6$ & $3,37 \pm 0,7$ & $117,5 \pm 39,7$ \\
5 & $6,2 \pm 1,8$ & $2,85 \pm 0,1$ & $154,5 \pm 77,1$ & $2,8 \pm 0,1$ & $143,0 \pm 0,0$ \\
Total & $5,89 \pm 3,4$ & $3,18 \pm 0,5$ & $112,50 \pm 57,0$ & $3,34 \pm 0,5$ & $162,3 \pm 90,1$
\end{tabular}

$*(\mathrm{P}>0,05)$ pelo teste de Tukey.
A duração do intervalo do parto ao primeiro serviço foi de $112,5 \pm 57,0$ dias, não diferindo $(P>0,05)$ entre as ordens de parição (Quadro 2). Esse valor foi considerado elevado, e provavelmente deve ter ocorrido em função da não detecção de estro, estro silencioso e estresse por calor, visto que, todos os animais aos 50 dias pós-parto, já tinham ovulado, e constatado a presença do corpo lúteo por exames ultrasonograficos.

0 período de serviço médio para concepção foi de $162,3 \pm 90,1$ dias, não diferindo $(\mathrm{P}>0,05)$ entre as ordens de parto (Quadro 2), embora este período de serviço seja elevado (162,3 dias), esse valor é ainda considerado satisfatório, pois ainda não compromete a performance produtiva do rebanho, resultando em intervalos de parto médio de 14,9 meses. Esses valores podem ser reduzidos, se obtiverem melhores valores para a detecção de estro, problema este que pode ser constatado pelo intervalo de parto ao primeiro estro pós-parto (5,89 semanas pós-parto) e intervalo do parto a primeira inseminação $(112,50 \pm 57,0$ dias) (Quadro 2). Tais observações corroboram os estudos de DuBois \& Williams (1980), Lara (1985) 98,1 $\pm 43,4$ dias, Van Werven et al. (1992) 112 dias, Kamimura et al. (1993) $74,0 \pm 13,9$ dias, Han \& Kim (2005), 108,0 dias, Drillich et al. (2006a) 93 a 108 dias e Kocamuftuoglue Vural (2008) $144,1 \pm 36,9$ dias.

No presente estudo, outros fatores que podem ter interferido no período de serviço foram, estros silenciosos pelos animais em tempo hábil após o período de espera voluntario e o elevado número de inseminações para concepção $(1,97 \pm 1,1$; Quadro 3). Entretanto, estudos anteriores com menor período de serviço tanto em vacas com ou sem infecção uterina, relatam índice de fertilização superiores (2,9 dose prenhes) aos obtidos o presente estudo (Barlund et al. 2008).

Os transtornos reprodutivos puerperais como retenção de placenta, infecção uterina verificados no presente estudo, não influenciaram o restabelecimento da ciclicidade ovariana $(\mathrm{P}>0,05)$, de modo que o intervalo do parto a primeira ovulação, entre animais que tiveram ou não retenção de placenta, ou ordem de parto ou categoria de vacas não diferiram $(P>0,05)$ entre as categorias (Quadro 2$)$.

As porcentagens de vacas que não tiveram transtornos reprodutivos, como retenção de placenta ou infecção uterina e haviam ovulado até a sétima semana após o parto (69,6\% para as primíparas e $77,1 \%$, para as pluríparas), sendo semelhantes aos estudos por Santos et al. (2002) obtiveram $74,5 \%$ dos animais ciclando até a sétima semana pós-parto, e as $78 \%$ das vacas do estudo de Senosy et al. (2009) que também retornaram a atividade ovariana no mesmo período.

Os índices reprodutivos de vacas que apresentam retenção de placenta geralmente são inferiores àqueles observados em vacas sem retenção de placenta. Mas no presente estudo essa diferença não foi observada devido ao diagnostico precoce e tratamento imediato dos animais que apresentaram retenção de placenta.

No presente estudo não houve relação da ordem de parto com ocorrência de retenção de placenta $(P>0,05)$ corrobarrando com estudos anteriores de Van Werven et al. 
(1992) e Kaneko et al. (1997) que não verificaram relação entre retenção de placenta e intervalo do parto à primeira IA. Entretanto, os referidos autores verificaram valores inferiores de intervalo do parto à 1 IA sendo respectivamente de 78 e 75 dias para primíparas e pluriparas de dois ou três partos, que apresentaram ou não retenção de placenta por mais de 12 horas e de $90,0 \pm 28,5$ e 83,0 $\pm 32,2$ dias para animais com ou sem retenção de placenta. Valores semelhantes foram registrados por Drillich et al. (2003) com intervalos do parto ao primeiro serviço de 75,2 e 81 dias, para animais que receberam diferentes tratamentos para retenção de placenta. No entanto, valores elevados de intervalo do parto ao primeiro serviço também foram registrados por Kocamuftuoglu \& Vural (2008), que registraram 117,0 e 91,7 dias, para animais com ou sem transtornos puerperais, respectivamente.

A taxa de gestação ao primeiro serviço foi de 43,6 \% (17/39), sendo que dos 17 animais que se tornaram gestantes após a primeira inseminação artificial,53,0 \% (9/17) eram animais que não tiveram retenção de placenta nem infecção uterina e somente 47,1\% (8/17) haviam apresentado infecção uterina (Quadro 4), e desses 8 animais, 17,6\% (3/8) apresentaram associação de retenção de placenta com infecção uterina.

Em rebanhos leiteiros de alta produção, a taxa de gestação ao primeiro serviço geralmente é inferior a $40 \%$, sendo necessárias mais de duas doses de sêmen para tornar a vaca gestante (Royal et al. 2000). No presente estudo a taxa de gestação ao primeiro serviço foi de 43,6\%. Possivelmente, essa taxa deve-se ao uso de sêmen de touros provados, ao diagnóstico e tratamento precoce da retenção de placenta, infecção uterina e cistos foliculares, antes mesmo dos animais apresentarem o primeiro estro pós-parto. Também se deve possivelmente, aos estros não observados, com o aumento do número de ciclos estrais antes da primeira inseminação artificial, proporcionando melhor ambiente uterino pós-parto, e maior probabilidade de se tornar gestante na primeira inseminação artificial ou por monta natural. Por tanto, a taxa de gestação poderiam ser melhores, caso a detecção de estro fosse mais eficiente.

No segundo grupo (Quadro 4), embora não tenha sido constatado diferença no percentual de fêmeas gestantes com relação ao grupo 1, os tratamentos das infecções uterinas também foram eficazes, porém a razão de maior número de inseminação/concepção se deve provavelmente ao

Quadro 3. Número de inseminação artificial para concepção (NAICONC)*, número de inseminação (NIA), números de ciclos para primeira inseminação artificial (NCL1IA)*, e diferença de escore de condição corporal do parto a concepção (DPCONC)* em vacas da raça Holandesa-PB, em diferentes ordens de parto (ORDPART)*

\begin{tabular}{ccccc}
\hline ORDPART & NIACONC & NIA & NCI1IA & DPCONC \\
\hline 1 & $2,18 \pm 1,6$ & $2,41 \pm 1,7$ & $5,12 \pm 0,1$ & $-0,15 \pm 0,5$ \\
2 & $1,87 \pm 1,0$ & $2,10 \pm 1,3$ & $5,15 \pm 2,7$ & $-0,57 \pm 0,4$ \\
3 & $2,14 \pm 1,1$ & $2,14 \pm 1,1$ & $5,0 \pm 2,4$ & $-0,12 \pm 0,4$ \\
4 & $1,50 \pm 0,6$ & $1,50 \pm 0,6$ & $4,25 \pm 1,5$ & $-0,37 \pm 0,8$ \\
5 & $2,0 \pm 0,0$ & $2,33 \pm 1,5$ & $4,0 \pm 0,0$ & $-0,75 \pm 0,0$ \\
Total & $1,97 \pm 1,1$ & $2,15 \pm 1,4$ & $4,9 \pm 2,1$ & $-0,15 \pm 0,5$
\end{tabular}

* $(\mathrm{P}>0,05)$ pelo teste $\mathrm{F}$. estresse calórico, problemas podais, partida do sêmen e de touro utilizado, manejo do sêmen e botijão, ou ate mesmo os diferentes inseminadores. Embora em outrosestudos (Drillich et al. 2003, Drillich et al. 2006b, Kocamuftuoglu \& Vural 2008, Tillard et al. 2008) tenham atribuido baixo índice de fertilidade ( 25 a $37 \%$ ) a presença de retenção de placenta ouinfecções uterina pós-parto, no presente estudo como mencionado anteriormente não se mostrou relacionado a estas patologías.

A média do número de serviço por concepção, número de inseminações artificiais, número de ciclos para a primeira inseminação artificial no pós-parto e a diferença de escore de condição corporal do parto até concepção não diferiram entre os valores médios nas ordens de parto (Quadro 3 ). Ao final do experimento, $76,4 \%$ das vacas encontravam-se gestantes.

Quadro 4. Taxa de gestação* em vacas com aptidão leiteira, de acordo com o número de serviço/prenhes e presença ou ausência de infecção uterina

\begin{tabular}{lccc}
\hline \multicolumn{1}{c}{ Grupo de aimais } & $\%(\mathrm{~N})$ & $\begin{array}{c}\text { Sem infecção } \\
(\%)\end{array}$ & $\begin{array}{c}\text { Com infecção } \\
(\%)\end{array}$ \\
\hline Gestantes ao primeiro serviço & $43,6(17 / 39)$ & $52,9(9 / 17)$ & $47,1(8 / 17)$ \\
Gestantes com mais de 1 Serviço & $56,4(22 / 39)$ & $82,0(18 / 22)$ & $18,0(4 / 22)$ \\
Total & $100,0(39)$ & $69,2(27 / 39)$ & $30,8(12 / 39)$
\end{tabular}

$\bar{*}(\mathrm{P}>0,05)$ pelo teste de Qui-Quadrado; $\mathrm{N}=$ número de vacas no grupo.

A média de serviços por gestação foi superior à reportada por Lara (1985) que foi de $1,7 \pm 1$ inseminações por gestação e aos de Kamimura et al. (1993) que foi de 1,4 $\pm 1,0$ inseminações por gestação, também empregando o manejo de free stall.

Esperava-se no presente estudo maior número de serviços por concepção dos animais com infecção uterina, em relação aos animais sem infecção uterina. Isto pelo fato do ambiente uterino ser inadequado após a instalação de processos infecciosos graves, o que influencia na implantação e manutenção da gestação (Fernandes et al. 2001, Kim \& Kang 2003), os valores considerados satisfatórios, provavelmente, isto se devem ao diagnóstico e tratamento precoce das infecções uterinas, levando uma rápida convalescência dos animais.

Transtornos puerperais como retenção de placenta, infecção uterina e doenças metabólicas são responsáveis pelo prolongamento do intervalo do parto ao primeiro serviço, mas no presente estudo, esses transtornos foram diagnosticados e tratados precocemente e não se mostraram relacionados ao período de serviço.

Foi registrada correlação do ECC ao parto e a incidência de retenção de placenta, tanto para primíparas e pluríparas com retenção de placenta, onde apresentaram maior ECC e peso ao parto.

As maiores porcentagens de incidências de retenção de placenta foram para as vacas com condição corporal média $(3,75)$ e menor porcentagem para os animais de ECC $(3,25-$ 3,5) (Quadro 5). Não foi observada retenção placentária para os animais com ECC baixa $(2,5)$, porém apenas dois animais apresentaram este escore da condição corporal ao parto. 
Quadro 5. Escore de condição corporal (ECC) e peso corporal ao parto em vacas da raça Holandesa $\mathrm{PB}$, de acordo com a ocorrência de retenção de placenta

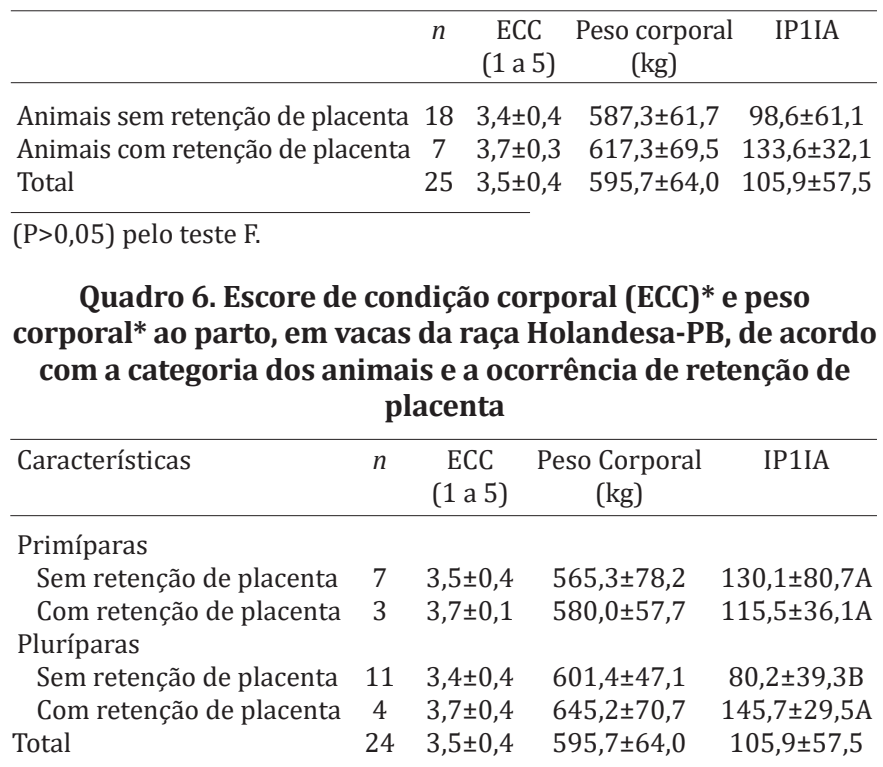

$*(\mathrm{P}<0,05)$ pelo teste $\mathrm{F}$.

No presente estudo, houve uma distribuição equitativa na frequência de retenção de placenta nos grupos de primíparas e pluríparas (Quadro 6).

No presente estudo somente os animais acima da segunda ordem de parição (pluríparas) demonstraram efeito negativo da retenção de placenta, apresentando maior intervalo de parto à primeira inseminação artificial (Quadro 6). Provavelmente se deve nas diferenças entre as frequências e maior peso ao parto nas vacas pluríparas, sem afetar o período de serviço.

Santos et al. (2002) notaram relação entre retenção de placenta e ECC ao parto, onde observaram maior incidência dessa patologia em vacas obesas. Contrário aos resultados de Markusfeld et al. (1987) que registraram maior risco de retenção de placenta em vacas com baixo ECC ao parto. Entretanto, os valores obtidos no presente estudo e os realizados por Santos (1996); Fernandes et al.(2001) mostram-se contrários, onde os autores não verificaram relação entre a ocorrência de retenção de placenta e a condição corporal ao parto.

Nos parâmetros produtivos, as médias de produção de leite total, dias de lactação e produção de leite diária estão sumariadas no Quadro 7, não sendo observada diferença

Quadro 7. Produção de leite total (PRODLTO), dias de lactação (DLACTA) e produção de leite diária (PRODLDI) e produção

de leite ajustado para 305 (PRODL305) em vacas da raça Holandesa-PB, de acordo com a ordem de parto (ORDPART)*

\begin{tabular}{ccccc}
\hline ORDPART & PRODLTO $(\mathrm{kg})$ & DLACTA $(\mathrm{kg})$ & PRODLDI (kg) & PRODL305(kg) \\
\hline 1 & $8330,4 \pm 4146,9$ & $412,0 \pm 84,6$ & $21,6 \pm 6,0$ & $7167,5 \pm 2172,5$ \\
2 & $6999,3 \pm 2030,0$ & $326,3 \pm 81,3$ & $21,8 \pm 5,6$ & $7090,5 \pm 1605,6$ \\
3 & $9717,0 \pm 2912,0$ & $381,0 \pm 87,1$ & $25,8 \pm 6,6$ & $8576,6 \pm 1790,9$ \\
4 & $8190,3 \pm 1588,4$ & $341,0 \pm 52,5$ & $24,0 \pm 1,3$ & $7541,0 \pm 690,5$ \\
5 & $8071,0 \pm 4142,8$ & $314,6 \pm 132,7$ & $25,0 \pm 4,0$ & $7635,0 \pm 2833,9$ \\
Total & $8000,4 \pm 3065,8$ & $358,3 \pm 90,9$ & $22,9 \pm 5,6$ & $7424,0 \pm 1877,8$
\end{tabular}

$*(\mathrm{P}>0,05)$ pelo teste de Tukey. entre os valores médios nas diferentes ordens de parto $(\mathrm{P}>0,05)$.

As médias de produção de leite total, dias de lactação e produção de leite diária, estão sumariadas no Quadro 8, não diferiram entres as vacas gestantes e não gestantes $(\mathrm{P}>0,05)$.

Os valores médios para características de produção entre as ordens de parto não diferiram $(p>0,05)$. Quando se analisou a produção ajustada para 305 dias, também não houve diferença entre os valores médios nas diferentes ordens de parto (Quadro 8). Isto reflete provavelmente no programa de melhoramento no qual o referido rebanho vem sendo submetido, onde os animais de reposição são previamente selecionados entre as bezerras nascidas no próprio rebanho.

No presente trabalho, apesar de não ter sido observado diferença entre produção de leite total, dias de lactação, produção de leite diária e na produção de leite ajustado para 305 dias de lactação nas diferentes ordens de partos (Quadros 7 e 8) foram observadas correlações desses parâmetros com alguns aspectos reprodutivos. A produção de leite acumulada até a concepção apresentou correlação com o intervalo de parto à primeira inseminação $(r=0,56)$, número de ciclos até a primeira inseminação $(\mathrm{r}=0,49)$, número de inseminações por concepção $(r=0,36)$ e com o período de serviço $(\mathrm{r}=0,84)$. A produção de leite diária apresentou correlação somente com número de inseminações à concepção $(\mathrm{r}=0,32)$. A produção de leite total apresentou correlação com intervalo do parto à primeira ovulação $(\mathrm{r}=0,38)$, intervalo de parto à primeira inseminação $(\mathrm{r}=0,50)$ e período de serviço $(\mathrm{r}=0,40)$.

Os dias de lactação apresentaram correlação com intervalo de parto a primeira inseminação $(\mathrm{r}=0,63)$, número de ciclos à primeira inseminação $(\mathrm{r}=0,35)$, número de inseminações à concepção $(\mathrm{r}=0,49)$ e com período de serviço $(\mathrm{r}=0,81)(\mathrm{p}<0,05)$. Já, quando se ajustou a produção de leite aos 305 dias de lactação, não se observou nenhuma correlação com os parâmetros reprodutivos $(p>0,05)$.

Com exceção das correlações verificadas com o período de serviço que se mostraram elevadas, e a relação entre dias de lactação com intervalo do parto à primeira inseminação que foi alta, as demais correlações foram medianas. Esta relação entre produção e reprodução registrada no presente estudo, e diante dos resultados observados para os parâmetros reprodutivos demonstram que a produção de leite não afetou a performance reprodutiva $(76,4 \%$ vacas gestantes e 1,97 doses/prenhes), sendo considerada satisfatória para o manejo em free stall.

No entanto, Lucy (2001) e Butler (2008) observaram redução da fertilidade com o aumento da produção de lei-

Quadro 8. Produção de leite total (PRODLTO), dias de lactação (DLACTA) e produção de leite diária (PRODLDI) em vacas da raça Holandesa-PB, gestantes ou não gestantes*

\begin{tabular}{lccc}
\hline \multicolumn{1}{c}{ Categ. } & PRODLTO $(\mathrm{kg})$ & DLACTA $(\mathrm{kg})$ & PRODLDI $(\mathrm{kg})$ \\
\hline Gestante & $7.907,5 \pm 2.815,8$ & $366,8 \pm 79,2$ & $22,2 \pm 5,2$ \\
Nãogestante & $8.588,8 \pm 4.656,3$ & $307,0 \pm 145,0$ & $27,4 \pm 6,3$ \\
Total & $8.000,4 \pm 3.065,8$ & $358,6 \pm 91,0$ & $22,9 \pm 5,6$
\end{tabular}

* $(\mathrm{P}>0,05)$ pelo teste $\mathrm{F}$. 
Quadro 9. Peso ao parto (PESPART), escore da condição corporal ao parto (ECCPART), intervalo parto a ovulação (IPOVU), escore da condição corporal a primeira ovulação (ECC10VU) e a diferença do escore da condição corporal a primeira ovulação (DP10VU), de acordo com a categoria animal $^{*}$

\begin{tabular}{lccccc}
\hline \multicolumn{1}{c}{ Categ. } & PESPART & ECCPART & IPOVU & ECC1OVU & DP1OVU \\
\hline Gestante & $590,3 \pm 67,9$ & $3,5 \pm 0,6$ & $5,8 \pm 3,6$ & $3,2 \pm 0,5$ & $-0,3 \pm 0,4$ \\
Nãogestante & $607,5 \pm 5,1$ & $3,4 \pm 0,5$ & $6,1 \pm 2,9$ & $3,1 \pm 0,5$ & $-0,3 \pm 0,5$ \\
Total & $594,4 \pm 65,1$ & $3,5 \pm 0,6$ & $5,9 \pm 3,4$ & $3,2 \pm 0,5$ & $-0,3 \pm 0,4$
\end{tabular}

$*(\mathrm{P}>0,05)$ pelo teste $\mathrm{F}$.

te em vacas da raça Holandês. A redução na performance reprodutiva é refletida pelo atraso na atividade ovariana pós-parto e/ou diminuição na taxa de concepção e falhas na detecção de estro, principalmente nas vacas de alta produção.

0 peso ao parto, escore da condição corporal ao parto, intervalo do parto a ovulação, escore da condição corporal a primeira ovulação e a diferença da condição corporal ao parto a primeira ovulação, não diferiram entres as categorias gestantes e não gestante (Quadro 9).

No trabalho de Butler e Smith (1989) observa-se que à medida que os animais perderam maior unidade de escore $(0,5 ; 0,5-1 ;>1)$ assim mesmo foi aumentando a tendência de dias para a primeira ovulação $(27,31$ e 42 dias), intervalo para o primeiro estro (48, 41 e 62 dias) e para primeiro serviço (68, 67 e 79 dias) e a porcentagem da taxa de concepção foi cada vez menor (65, 53 e 17\%). Por tanto, no presente estudo o parâmetro reprodutivo afetado foi período de serviço, provavelmente devido a falhas na detecção de estro, constatado pela presença do corpo lúteo cíclico por meio de exames ultrasonográficos (Quadro 2).

\section{CONCLUSÕES}

A detecção de estro se mostra o maior fator limitante para alcançar melhores índices de eficiência reprodutiva e o acompanhamento no pós-parto imediato é fundamental para o diagnostico e tratamento de desordens reprodutivas puerperais, evitando o aumento do período de serviço e comprometimento dos índices reprodutivos e produtivos do rebanho.

O monitoramento do escore da condição corporal esta diretamente relacionado com a maximização da performance reprodutiva na pecuária leiteira e prevenção de transtornos reprodutivos, metabólicos e hormonais, de modo que, quando isso é alcançado, a produção de leite não afeta a performance reprodutiva do rebanho em manejo de free stall.

Agradecimentos.- Os autores agradecem ao Programa de Pós-graduação do Departamento de Veterinária da Universidade Federal de Viçosa (UFV) e ao contribuição das empresas de financiamento CAPES, CNPq e FAPEMIG, bem como ao Setor de Gado de Leite da UFV pelo apoio a pesquisa.

\section{REFERÊNCIAS}

Barlund C.S., Carruthers T.D., Waldner C.L. \& Palmer C.W. 2008. A comparison of diagnostic techniques for postpartum endometritis in dairy cattle. Theriogenology 69:714-723.

Butler W.R. 2006. Efeito do balanço energético negativo na fertilidade de vacas leiteiras. Anais X Curso Novos Enfoques na Produção e Reprodução de Bovinos, Uberlândia, MG.

Butler W.R. 2008. Produção de leite, balanço energético negativo e fertilidade em vacas leiteiras. Anais XII Curso Novos Enfoques na Produção e Reprodução de Bovinos, Uberlândia, MG, p.26-36.

Butler W.R. \& Smith R.D. 1989. Interrelationships between energy balance and postpartum reproductive function in dary cattle. J Dairy Sci. 72(3):767-783.

Campos O.F. \& Lizieire R.S. 1998. Estratégias para obtenção de fêmeas de reposição em rebanhos leiteiros. Anais do X Simpósio sobre Produção Animal, Piracicaba, SP, p.215-255.

Drillich M., Pfutzner A., Sabin H.J., Sabin M. \& Heuwieser W.2003. Comparison of two protocols of retained fetal membranes in dairy cattle. Theriogenology 59:951-960.

Drillich M., Mahlstedt M., Reichert U., Tenhagen B.A. \& Heuwieser W. 2006a. Strategies to improve the therapy of retained fetal membranes in dairy cows. J. Dairy Sci. 89:627-635.

Drillich M., Reichert U., Mahlstedt M. \& Heuwieser W. 2006b. Comparison of two strategies for systemic antibiotic treatment of dairy cows with retained fetal membranes: preventive vs. selective treatment. J. Dairy Sci. 89:1502-1508.

Dubois P.R. \& Williams D.J. 1980. Increased incidence of retained placenta associated with heat stress in Dairy cows. Theriogenology 13:115-121.

Fernandes C.A.C., Costa D.S. \& Viana J.H.M. 2001. Impacto da retenção de placenta sobre a performance reprodutiva de vacas leiteiras. Revta Bras. Reprod. Anim. 25:26-30.

Gearhart M.A., Curtis C.R., Erb H.N., Smith R.D., Sniffen C.J., Chase L.E. \& Cooper M.D. 1990. Relationship of changes in condition score to cow health in Holsteins. J. Dairy Sci. 73:3132-3140.

Han Y.K. \& Kim I.H. 2005. Risk factors for retained placenta and the effect of retained placenta on the occurrence of postpartum diseases and subsequent reproductive performance in dairy cows. J. Vet. Sci. 6:53-59.

Horta A.E.M. Fisiologia do puerpério na vaca. 1995. Anais das VIII Jornadas Internacionales de Reproducción Animal, Santander, Santander Espanha: p.73-84.

Kaneko J.J., Harvey J.W. \& Bruss M.C. 1997. Clinical Biochemistry of Domestic Animals. 5th ed. Academic Press, San Diego.

Kamimura S., Ohgi T., Takahashi M. \& Tsukamoto T. 1993. Postpartum resumption of ovarian activity and uterine involution monitored by ultrasonography in Holstein cows. J. Vet. Med. Sci. 55:643-647.

Kim I.H. \& Kang H.G. 2003. Risk factors for postpartum endometritis and the effect on reproductive performance in dairy cows in Korea. J. Reprod. Develop. 49:485-491.

Kocamuftuoglu M. \& Vural R. 2008. The evaluation of postpartum period in dairy cows with normal and abnormal periparturient problems. Acta Veterinaria, Beograd, 58:75-87.

Lara J.R.L. 1985. Alguns aspectos reprodutivos de um rebanho leiteiro no período pós-parto. Dissertação de Mestrado em Medicina Veterinária, Escola de Veterinária, Universidade Federal de Minas Gerais, Belo Horizonte. 58p.

Lucy M.C. 2001. Reproductive loss in high- producing dairy cattle: where will it end? J. Dairy Sci. 84:1277-1291.

Markusfeld 0. 1987. Periparturient traits in seven dairy herds: incidence rates association with parity, and interrelationship among traits. J. Dairy Sci. 70:158-166.

Marques Júnior A.P. 1993. Fisiologia do puerpério na vaca. Revta. Bras. Reprod. Anim. 4(Supl):58-69.

Royal M., Mann G.E. \& Flint A.P.F. 2000. Strategies for reversing the trend towards subfertility in dairy cattle. Vet. J. 160:53-60.

Santos R.L. 1996. Condição corporal ao parto e tempo requerido para liberação da placenta em vacas leiteiras. Arq. Bras. Med. Vet. Zootec. 48:235-238.

Santos R.M., Vasconcelos J.L.M., Souza A.H., Meneghetti M. \& Ferreira J.R. 2002. Efeito da aplicação de prostaglandina (PGF2alfa) no pós-parto imediato sobre a incidência de retenção de placenta em vacas de leite. Arq. Bras. Med. Vet. Zootec. 54:29-34. 
Santos R.M. 2005. Efeito da quantidade de concentrado da dieta de vacas holandesas não lactantes na progesterona plasmática, composição do fluído folicular e produção de prostaglandina pelo endométrio. Tese de Doutorado, Faculdade de Ciências Agrárias e Veterinária, Universidade Estadual Paulista, Jaboticabal, SP.

Senosy W.S., Uchiza M., Tameoka N., Izaike Y. \& Osama T. 2009. Association between evaluation of the reproductive tract by various diagnostic tests and restoration of ovarian cyclicity in high-producing dairy cows. Theriogenology 72:1153-1162.

Short R.E., Bellows R.B., Staigmiller J.G., Berardinelli J.G. \& Custer E.E. 1990. Physiological mechanismiscontroling anestrus and fertility in pospartum anestrus beef cattle. J. Anim. Sci. 68:799-816.

Tillard E., Humblot P., Faye B., Lecomte P., Dohoo I. \& Bocquier F. 2008. Postcalving factors affecting conception risk in Holstein dairy cows in tropical and sub-tropical conditions. Theriogenology 69:443-457.

Trimberg W. \& Davis H.P. 1943. Conception rate in dairy cattle by artificial insemination at various stages of estrus. Research Bulletin of the University of Nebraska, Lincoln. 14p.

Van Werven T., Schukken Y.H., Lloyd J., Brand A., Heeringa H.T. \& Shea M. 1992. The effects of duration of retained placenta on reproduction, milk production, postpartum disease and culling rate. Theriogenology 37:1191-1203.

Vanholder T., Leroy J.L.M.R., Dewulf J., Duchateau L., Coryn M., Kruif A. \& Opsomer G. 2005. Hormonal and metabolic profiles of high-yielding dairy cows prior to ovarian cyst formation or first ovulation postpartum. Reprod. Domestic Anim. 40:460-467.

Yavas Y. \& Walton J.S. 2000. Induction of ovulation in postpartum suckled beef cows: a review. Theriogenology 54:1-23. 